\title{
Hospital Utilization and Mortality in Transition-aged Patients with Sickle Cell Disease in the United States
}

\author{
Titilope Fasipe ${ }^{1}$, Deepa Dongarwar ${ }^{1}$, Ria Brown ${ }^{1}$, Elyse Lopez ${ }^{1}$, Megan Abadom ${ }^{1}$, Donald \\ Mahoney $^{1}$, and Hamisu Salihu ${ }^{1}$ \\ ${ }^{1}$ Baylor College of Medicine
}

September 11, 2020

\begin{abstract}
Background: Sickle cell disease (SCD) is a genetic blood disorder that results in vaso-occlusive events and shortened lifespan. Medical advancements have led to a decrease in childhood mortality in SCD, but the transition period is associated with poor outcomes. We analyzed recent US hospitalizations and mortality trends in the transition-aged population and evaluated for differences between patients with and without SCD. Methods: Nationwide Impatient Sample (NIS) database was utilized to analyze hospitalizations among individuals aged 16-24 years from 2003-2017. Diagnoses were coded using ICD-9-CM and ICD10-CM. Statistical analyses included: bivariate analyses to assess the association between socio-demographic characteristics, joinpoint regression analysis to describe trends in mortality rates, average annual percent change (AAPC) measures were calculated, and adjusted survey logistic regression to assess the association between patient characteristics and in-hospital mortality. Results: Our data captured 37,344,532 total patients between the age of 16 and 24 who were hospitalized during 2003-2017. Patients with SCD comprised of $1.20 \%$ of population with a significant $+3.2 \%$ AAPC in hospitalizations. When comparing SCD to non-SCD hospitalization trends, we observed differences in gender, against-medical-advice discharge status, zip code income, and payer. SCD in-hospital mortality rates showed a non-significant $-0.7 \%$ AAPC. However, in contrast to non-SCD patients, the odds ratios for in-hospital mortality significantly increased with age for SCD patients. Conclusion: During the transition period, SCD patients have an extremely increased likelihood of mortality. Proposed interventions to address this disparity must include targeting social determinants of health.
\end{abstract}

\section{Methods}

We analyzed hospitalization records from January 1, 2003 through December 31, 2017 that were contained in the Nationwide Inpatient Sample (NIS). The NIS datasets constitute the largest all-payer, publicly available inpatient database in the US and are made available by the Healthcare Cost and Utilization Project (HCUP). Although the systematic sampling process used to select the hospitalizations to be included in the NIS has changed over time, the result is an approximate $20 \%$ sample of hospital discharges from participating states. The sampling strategy ensures that hospitalizations in the NIS are representative of the population on important factors including month of admission, primary reason for hospitalization, and hospital size, location, ownership, and teaching status. The NIS contained approximately seven million inpatient hospitalizations each year (35 million when weighted) from around 47 participating states.

Our study sample included hospitalizations among transition-aged patients within the age range of 16 to 24 years. Diagnoses and procedures are coded using International Classification of Diseases, Ninth Revision, Clinical Modification (ICD-9-CM) diagnosis codes till the 3rd quarter of 2015, after which HCUP transitioned to ICD-10-CM format. To assess the study's primary exposure, we first scanned the diagnosis codes (the principal diagnosis and up to 29 secondary diagnoses) in each patient's discharge record for an indication of SCD (ICD-9-CM - 282.41, 282.42, 282.61, 282.62, 282.63, 282.64, 282.60, 282.68, 282.69; ICD-10-CM D57.4x, D57.0x, D57.2x, D57.1x, D57.8x). These sickle cell-related encounters were then sub-divided into 
four mutually exclusive categories based on ICD-9-CM and ICD-10-CM codes: HbSS (ICD-9-CM - 282.61, 282.62; ICD-10-CM - D57.0x), HbSC (ICD-9-CM - 282.62, 282.63; ICD-10-CM - D57.2x), HbS/ $\beta$-thalassemia (ICD-9-CM - 282.41, 282.42; ICD-10-CM - D57.4x), and 'Other SCD' (ICD-9-CM - 282.60, 282.68, 282.69; ICD-10-CM - D57.1x, D57.8x)

The covariates in this study included individual-level sociodemographic and hospital characteristics. We categorized patients' ages in groups of 16 to 18 years, 19 to 21 years and 22 to 24 years. Ethnicity was first stratified on the basis of reported ethnicity (Hispanic, non-Hispanic); and the non-Hispanic group was subdivided (White, Black, or other). The primary payer for the hospitalization was classified into Medicare, Medicaid, private, self-pay and other (including under/uninsured). As a proxy for socioeconomic status, the Healthcare Cost and Utilization Project provides zip-code-level estimates of median household income, grouped into quartiles based on the patient's residence. Hospital factors included census region (Northeast, Midwest, South, West), bed size (small, medium, large), and type (rural, urban-nonteaching, urban-teaching).

Descriptive statistics were utilized to represent the prevalence of SCD among all transition-aged hospitalizations and to demonstrate the prevalence of sub-types of SCD among all SCD hospitalizations for each year of the study period. We conducted bivariate analyses to assess the association between socio-demographic characteristics of the patients and SCD and its sub-types. Furthermore, to describe the trends in rates of in-hospital mortality among patients with SCD over the study period, we conducted joinpoint regression analysis, which is a statistical modeling approach specifically designed to evaluate and describe the extent to which the rate of a condition changes over time. The model first fits the annual rates of outcome of interest (i.e. in-hospital mortality) to a model with the minimum number of joinpoints (zero), suggesting that a straight line and single trend best fits the annual prevalence data. Then, more joinpoints are added iteratively to test the statistical significance of the various models using the Monte Carlo permutation method. Once the final (best-fitting) model with the optimal number of joinpoints has been selected, each joinpoint represents a statistically significant change in the trend, and each distinct trend is characterized using an annual percent change (APC) measure and its 95\% confidence interval (CI). Lastly, we conducted adjusted survey logistic regression model to assess the association between various patient characteristics and in-hospital mortality among patients with and without a diagnosis of SCD. Analyses were performed using R (version 3[?]6[?]1) and RStudio (Version 1[?]2[?]5001) and the Joinpoint Regression Program, version 4.7.0.0 (National Cancer Institute); we assumed a 5\% type I error rate for all hypothesis tests (two-sided). This study was deemed exempt by the IRB of Baylor College of Medicine as the study was performed on secondary de-identified data.

\section{Results}

Our data captured 37,344,532 patients between the ages of 16 and 24 who were hospitalized between 2003 and 2017. Patients with SCD accounted for 1.2\% (446,503) of total hospitalizations over the 15-year period (Supplemental Table 1). A temporal look at hospitalizations revealed an upward trend in the annual percentage of sickle cell admissions (Figure 1). Further analysis found a statistically significant Average Annual Percent Change (AAPC) of $+3.2 \%$ (Figure 1 and Supplemental Table 1).

The socio-demographic characteristics of patients with SCD in comparison to those without SCD are displayed in Table 1. During the study period, an increase in hospitalizations was noted with increasing age in both patient groups. In SCD, 22-24-year-olds accounted for $42.9 \%$ of total hospitalizations, whereas 16 18-year-olds only accounted for $22.9 \%$ (Table 1 ). When classified by gender, the distribution of non-SCD hospitalizations was comprised of majority female patients. On the other hand, female patients accounted for only $54.9 \%$ of SCD hospitalizations. The vast majority of patients with SCD were Non-Hispanic (NH) Black, with $81.5 \%$ of all patients with SCD identifying as such.

Among discharge outcomes, $92 \%$ of sickle cell admissions resulted in routine discharge, $2.1 \%$ were transferred, and $0.2 \%$ resulted in death; trends were similar in the non-SCD admissions (Table 1). Interestingly, patients with SCD were discharged from the hospital against medical advice (DAMA) 3.7\% of time, whereas DAMA 
only accounted for $1.2 \%$ of non-SCD discharge status (Table 1 ).

Regarding zip code income, nearly half $(48.5 \%)$ of all hospitalized patients with SCD fell into the lowest quartile. Patients with SCD were also more likely to use public insurance than private insurance, with $70.4 \%$ of all patients with SCD using either Medicaid or Medicare (Table 1).

Figure 2 depicts the temporal trend of in-hospital mortality rates for patients with SCD. While crude rates exhibited considerable variation year-by-year, further examination using joinpoint regression analysis revealed an AAPC of $-0.7 \%$, however this did not achieve statistical significance.

Table 2 further expounds upon potential associations between socio-demographic characteristics and inhospital mortality rates of transition-aged patients both with and without SCD. The most prominent finding was an association between increasing age and increased in-hospital mortality in patients with SCD. When using the 16-18-year-old subgroup as a reference, analysis showed an odds ratio (OR) of 2.09 for the 19-21year-old subgroup $(\mathrm{p}<0.0001)$ and 2.71 for the 22-24-year-old subgroup $(\mathrm{p}<0.0001)$. In contrast, this trend was not exhibited in the non-SCD group, with odd ratios of approximately 1 for each of the aforementioned subgroups (Table 2). In patients with SCD, no statistically significant difference in mortality was noted in respect to race (reference group $=$ NH-Black, Table 2). Female patients had a lower likelihood of mortality both in non-SCD and SCD groups, with an OR of 0.20 and 0.57 respectively (each p $<0.0001$, Table 2 ).

Within the SCD group, there were no observed significant differences in mortality between income quartile (Table 2). Among primary payer types, patients with SCD with private insurance were 1.39 times as likely to suffer in-hospital mortality $(\mathrm{p}=0.05)$, and those with self-pay were 1.53 times as likely $(\mathrm{p}=0.04)$ in comparison to those with Medicaid (Table 2).

\section{Discussion}

Despite the evidence for increased hardships and hospitalizations for transition-aged patients with SCD, we lack recent, nationwide studies pertaining to the trends in this population's mortality. Previous studies are now dated or had a narrow emphasis, focusing on specific demographics such as geographic regions. We thus embarked on a comprehensive approach to examine recent, national data regarding transitionaged hospitalizations in the SCD population. The Healthcare Cost and Utilization Project (HCUP)'s 2019 Statistical Brief \#251 reported that 18-34 year-old patients comprised the majority (50.7\%) of SCD stays between 2000-2016, a vast difference when compared to non-SCD admissions in which 18-34 year-olds only made up 16.3\%. However, in focusing on ages 16-24 specifically, our results did not show a difference in the portion of transition-age hospitalizations between SCD and non-SCD cohorts (Table 1). The HCUP brief also highlighted various socio-economic differences between SCD and non-SCD admissions. Correspondingly, we noted transition-aged patients with SCD were more likely to have public insurance, be in the lower zip income quartile and have a discharge status of DAMA (Table 1).

Furthermore, we were interested in the mortality risk among different SCD transition-age cohorts. We were astonished to see that in comparison to teens aged 16-18, the risk in mortality was 2 -fold higher for those aged 19-21, and nearly 3-fold higher for ages 22-24 (Table 2). These findings corresponded with 1999-2009 data obtained by Hamideh et al which demonstrated a sharp increase in the morality rate in patients with SCD between 15-19-years-old to 20-24-years-old.

An additional finding pertained to mortality differences based on documented sex. Female patients constituted a slight majority of patients hospitalized with sickle cell disease (Table 1), however, they were less likely to suffer in-hospital mortality when compared to their male counterparts (Table $2 ; \mathrm{p}<0.0001$ ). These results align with a previous study which determined that pediatric male patients with SCD have a worse, more aggressive clinical course than females and an increased morbidity.

Limitations of our study are related to the nature of database utilization; specifically, the absence of granular data and the inability to track a patient over time. Due to this, cause of death and other information regarding clinical course is unobtainable. Furthermore, the structure of the NIS data was confined to inpatient admissions only and did not contain ED visits, outpatient visits or urgent care visits. Despite 
some limitations, our study was highly powered in detecting differences in hospitalizations and mortality associated with SCD due to the large sample size. The NIS data was composed of weighted national samples, representative of the entire population rendering the findings in this study to be generalizable.

Several groups have underscored the vulnerability of patients with chronic diseases during the transition period; the consensus is that this period is plagued by social, psychological, and physiological hardships. For patients with SCD in particular, these challenges exist in a sort of cyclic nature, where increased pain episodes and fatigue lead to anxiety and depression, decreasing a patient's health-related quality of life and in turn further exacerbating the fatigue and pain episodes. These pain episodes, or vaso-occlusive crises, are the leading cause of SCD admissions, and serve as a primary risk factor for potential life-threatening events.

Our study has demonstrated the necessity for multifaceted improvements in the process of transitioning our pediatric patients with SCD to adult care. A 2009 survey showed that the vast majority of transitioning patients with SCD did not feel ready to transition, with only $34 \%$ of participants knowing which hospital they planned to transition to. Thus highlighting the importance of addressing barriers to transition readiness. A proposed solution to this problem has been the implementation of SCD transition programs and centers, however the efficacy and sustainability of these programs is still being monitored. Additionally, in a 2014 study regarding the risk factors for a successful transition, Andemariam et al. found that a greater distance between transition centers and patients with SCD was associated with unsuccessful transitioning. This underscores the importance of accounting for social determinants of health when addressing the transition process.

A further barrier to successful transition is a lack of knowledgeable providers. In a 2011 study that surveyed pediatric providers at several SCD centers, Sobota et al. found that only $60 \%$ of centers sent their patients to hematologists specializing in adult SCD and noted that some centers sent their patients to internist instead. Providers also noted that difficulty in finding adult SCD physicians was a large barrier for transition. This corresponds with evidence that transition-aged patients are less likely to receive disease-modifying therapies following childhood.

The final point is that age should be taken into consideration for transition. Andemariam et al. also found that an older age at the time of initiating the transition process correlated with unsuccessful transition (2014). Coupled with our findings of higher mortality with increasing transition age, it is imperative that transition readiness education begin in early adolescence.

\section{Conclusion}

Despite more than two decades of improved overall health care outcomes for patients with SCD, the transition from pediatric to adult health care continues to be associated with adverse health outcomes. Advancements in the medical management of SCD have resulted in a growing population of young adults living with SCD. Our analysis noted that all young adult patients had increased hospitalizations with age, however, only in the sickle cell cohort was there an increase in mortality with increasing age. Specifically, we have shown that mortality between young adults is $2-3 \mathrm{x}$ higher than in older teens. This highlights the necessity of maximizing disease-modifying therapies and engaging in transition-readiness programs that target social determinants of health.

\section{Conflict of Interest:}

TF is a consultant for Novartis and has provided education to Global Blood Therapeutics' staff.

\section{Acknowledgements:}

Research funding support was provided by the U.S. Department of Health and Human Services and Health Resources and Services Administration for Baylor College of Medicine Center of Excellence in Health Equity, Training, and Research (Grant No: D34HP31024).

Authorship contributions: Concept and design: TF, DD, and HMS. Collection and assembly of data: TF, DD, HMS. Data analysis and interpretation: all authors. Manuscript writing: all authors. Manuscript 
editing and final approval: all authors.

\section{References}

\section{Figure legends}

Figure 1: Temporal trends in hospitalization rates of any sickle cell disease among transition aged patients - 2003-2017. AAPC: average annual percentage change. ${ }^{*} p$-value $<0.05$.

Figure 2: Rates of in-hospital mortality among transition-aged patients with sickle cell disease. AAPC: average annual percentage change.

Supplemental Table 1: Prevalence of sickle cell disease and its sub-types among hospitalizations in the transition-aged population. SCD: sickle cell disease. AAPC: average annual percentage change. ${ }^{*} p$-value $<0.05$.

\section{Hosted file}

Table 1.docx available at https://authorea.com/users/356315/articles/479238-hospitalutilization-and-mortality-in-transition-aged-patients-with-sickle-cell-disease-in-theunited-states

\section{Hosted file}

Table 2.docx available at https://authorea.com/users/356315/articles/479238-hospitalutilization-and-mortality-in-transition-aged-patients-with-sickle-cell-disease-in-theunited-states

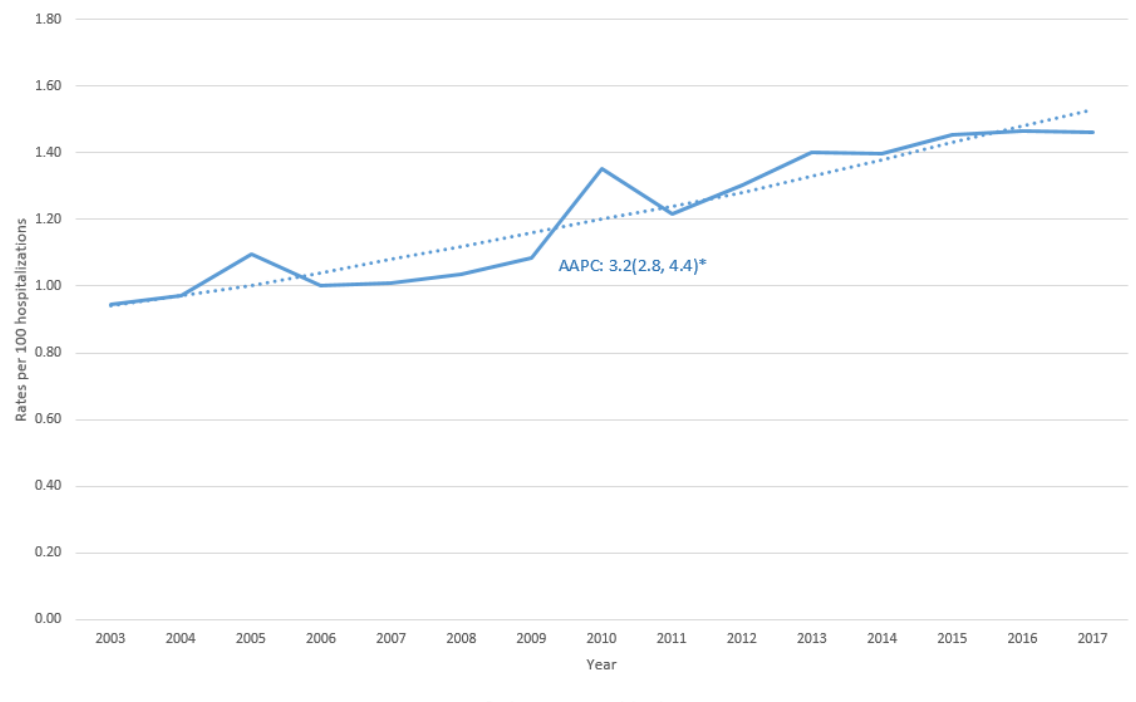




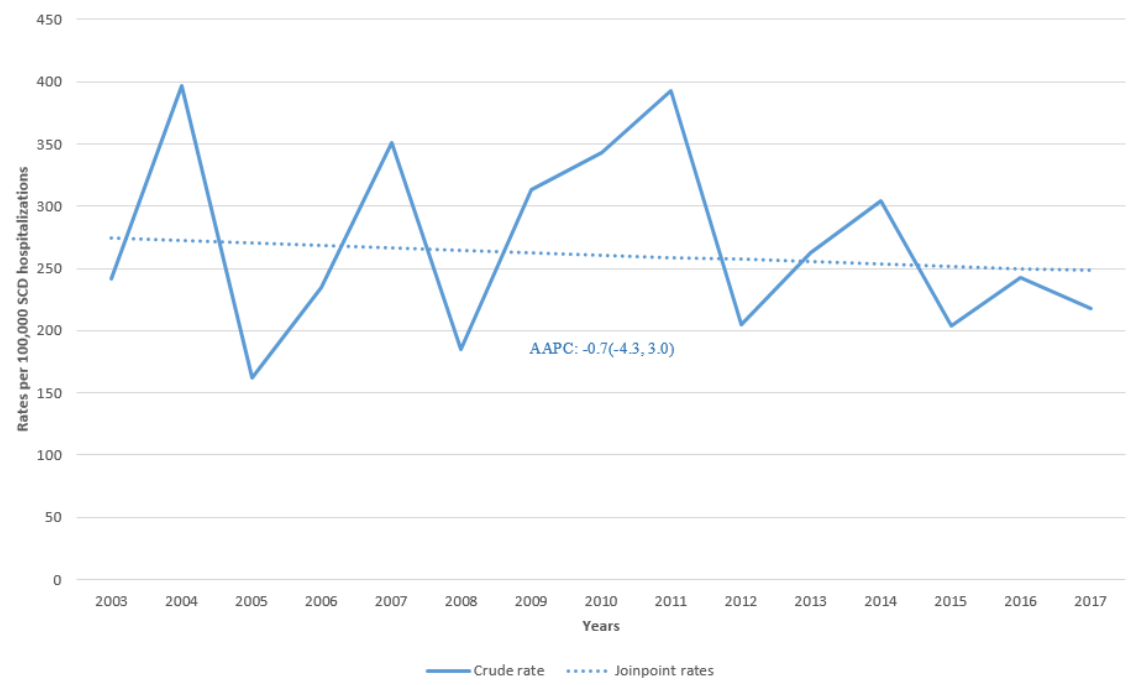

